\title{
PATHOLOGICAL INVESTIGATION OF MAREK'S DISEASE IN A PULLET FARM OF BANGLADESH AND DETECTION OF MAREK'S DISEASE VIRUS BY PCR
}

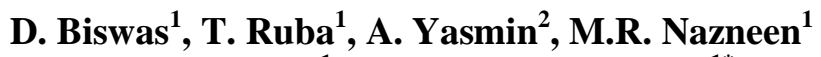 \\ M.M. Hossain ${ }^{1}$ and M.A.H.N.A. Khan ${ }^{1 *}$ \\ ${ }^{1}$ Department of Pathology, Faculty of Veterinary Science, Bangladesh Agricultural University, \\ Mymensingh-2202, Bangladesh \\ ${ }^{2}$ Department of Pathobiology, Faculty of Veterinary and Animal Science, Bangabandhu Sheikh Mujibur \\ Rahman Agricultural University, Salna, Gazipur, Bangladesh
}

\begin{abstract}
Marek's disease (MD) is a lymph proliferative disease of chickens, characterized by progressive emaciation, morbidity and mortality. The causative agent is a cell associated oncogenic alpha-herpes virus. This study investigated an outbreak of MD in a pullet farm ( $N=2200)$ of Ramu Upazilla, Cox's bazaar during May 2016 vaccinated against MD. The infectivity was reported on day 45 of age. Birds $(\mathrm{N}=10)$ submitted to diagnose disease at necropsy in the Department of Pathology, Bangladesh Agricultural University showed prominent keel bone, asymmetric progressive paralysis of one or both of the legs and wings. At necropsy, the skeletal muscle appeared thinner and there was enlargement of liver, spleen, kidney and sciatic nerve. Impression smears prepared from the liver showed huge infiltration of lymphocytes. Sections of heart, lungs, liver, kidney, nerve, skin and spleen were stained with hematoxylin and eosin showed wide spread infiltrations and accumulation of lymphocytes. Lymphocytic infiltration was seen in the skin, nerves and all visceral organs and showed combined infectivity due to visceral and classical forms. The etiology of MD was confirmed by using polymerase chain reaction (PCR) targeting fragment of Meq gene of very virulent plus or very virulent MDV1. Results of PCR showed amplification of $317 \mathrm{bp}$ fragment of Meq gene suggestive for infectivity due to MDV1GA (Md/5) strain. It requires isolating viruses in culture to test further for its virulence and pathotype in vivo. Sequencing and phylogenetic analysis of Meq gene may unveil the pathotype of the virus involved.
\end{abstract}

Keywords: Marek's disease, pullet, PCR, Meq gene, MDV1GA strain

\footnotetext{
* Corresponding author mail: hadi.khan@bau.edu.bd
} 


\section{INTRODUCTION}

Marek's disease (MD) in adult cockerels with leg paralysis was first described in 1907 by Jozsef Marek in Hungary (Marek, 1907). The name "Marek's disease" was first proposed by Biggs in 1961 and was regarded as synonymous with "fowl paralysis" or "neurolymphomatosis" (Pappenheimer et al., 1929). Due to association of lymphoid tumors in the peripheral nerves and visceral organs, the disease wasnamedas "neuro-lymphomatosisgallinarum" (Pappenheimer et al., 1929). The disease is caused by viruses, lymphoproliferative in nature, widely prevalent in commercial flock and causes variable rate of morbidity and mortality. Confirmatory diagnosis of lymphoproliferative diseases in poultry is often difficult. Three main classes of virus causing neoplasms of lymphatic tissues in poultry area) Marek's disease virus (MDV), a herpes virus b) Avian leukosis virus (ALV) and a retrovirus c) Reticuloendotheliosis virus (REV). Commercial chickens are the species most commonly affected with these viruses and neoplasms. Turkeys and quails can suffer from neoplasms caused by MDV and REV. Reticuloendotheliosis virus also causes neoplasms in geese and Muscovy ducks, pheasants and partridges (Payne and Venugopal, 2000). Marek's disease in chickens is a common malady in layer or elderly chickens and is characterized by the formation of lymphoma in different visceral organs and infiltration and proliferation of lymphoid cells into peripheral nerves (Quere, 1992).

Marek's disease virus induces T-cell lymphoma in chickens and is a member of the Alphaherpes virinae sub family of Herpes viridae. There are three serotypes of MDV: a) serotype 1 includes all pathogenic or oncogenic strains; b) serotype 2 includes naturally occurring non-pathogenic strains; and c) serotype 3 consists of turkey herpes virus (HVT), an oncogenic, MDV-related virus isolated from turkeys that has been widely used for immunization against Marek's disease (Zhang et al., 2014). Blymphocytes are the principal cells first targeted by the virus in a lytic fashion (Haq

et al., 2013). The early cytolytic events contributed in atrophy of the bursa of Fabricius and thymus, leading to severe debilitation of the immune system and marked immunosuppression. The systemic infectivity spread viruses to the feather follicle epithelium, from where viruses shed in the environment and infect other birds (Heidari et al., 2016).

Before the introduction of vaccination of commercial flocks in 1971, MD was a major global issue in chickens. Vaccination dramatically reduced losses, but the disease remained one of the significant burdens for poultry industry, particularly because of the periodic appearance of new strains of MDV against which existing vaccines provide suboptimal protection. This has required the continued development of new vaccines and vaccination strategies (Payne and Venugopal, 2000). The diagnosis of infection with MDV is usually made by observing clinical signs (OIE 2010), isolation and identification of MDV from the infected tissues in cell culture or identification of the infected cells by immune histochemistry (De Laney et al., 1998). 
However, recently the polymerase chain reaction (PCR) has appeared as a quick and sensitive method of diagnosis of MDV in a variety of tissues and materials (Handberg et al., 2001). Real-time polymerase chain reaction (RT-PCR) assays were developed simultaneously to measure MDV genome number in chicken tissues and environmental samples (Baigent et al., 2006; Islam et al., 2004). The pullet flock investigated in this study was vaccinated earlier against MD but the flock had lymphoproliferative lesions and suspected as a case of MD. The extensive clinical signs, gross and histopathology and detection of $\mathrm{Meq}$ gene of MDV by polymerase chain reaction was, therefore, carried out to identify the cause of illness and death of infected chicken.

\section{MATERIALS AND METHODS}

\section{Disease investigation}

This investigation was carried out in the Department of Pathology, Faculty of Veterinary Science, Bangladesh Agricultural University (BAU), Mymensingh. A pullet farm of Cox's bazar district had 2200 chickens that showed progressive weight loss, higher rate of morbidity and mortality. There was paralysis of legs and wings and the disease was suspected as a case of MD. Extensive investigation was, therefore, carried out for confirmatory detection of the specific cause of illness. A total of 10 pullets were investigated and samples were collected at necropsy during May 2016. Systemic dissection and analysis were carried out on to the liver, sciatic nerve, feather follicles, intestine, skeletal muscles, kidneys, spleen, heart, lungs, and skin to unveil the cause of illness.

\section{Histology of tissue sections}

The collected tissue samples were preserved in $10 \%$ buffered neutral formalin and processed for histopathological investigation. Thin sections of liver, spleen, heart, kidney, skeletal muscle, feather follicles, sciatic nerve, intestine and lungs were embedded in paraffin, sectioned at $4-5 \mu \mathrm{m}$ using a microtome, deparaffinized in xylene and stained with H\&E (Luna, 1968). The stained sections on to the slides were mounted using DPX, air dried and studied under low and high power microscopic fields.

\section{Giemsa staining of smears}

The liver of sick and dead pullets was cut into fine pieces, soaked with paper towel and impression smears were made onto the clean slides. The slides were air dried, fixed in ice cool absolute methanol for 45 mins. Smears were also taken from the venous blood onto clean slides, fixed for $45 \mathrm{mins}$ in ice cool methanol and air dried. The slides were stained with Giemsa's for 45 mins (Luna, 1968), washed in distilled water, air dried and examined under 100x microscopic field. Special emphasis was given to identify the type of mononuclear cells distributed in the smears. 


\section{Detection of Meq gene of MDV by PCR}

Sections of liver, spleen, heart, kidney, skeletal muscle, feather follicles, sciatic nerve, intestine and lungs were preserved at $-20^{\circ} \mathrm{C}$. The tissues were used to extract genomic DNA and PCR detection of $M e q$ gene of MD viruses. Traditional method was used to extract DNA ( $\mathrm{N}=10)$ from tissues of suspected pullets. Briefly, about 200 $\mathrm{mg}$ of individual tissue was macerated on sterile mortar and pestle while still in frozen. The crushed samples were transferred into the eppendorf tube containing $600 \mu \mathrm{l}$ cell lysis buffer and incubated at $56^{\circ} \mathrm{C}$ for an hour. The tissue suspension was then centrifuged at $5000 \mathrm{~g}$ for $10 \mathrm{mins}$. The supernatant was collected in a fresh tube, $3 \mu \mathrm{l}$ of RNase solution was added to the nuclear lysate and mixed by inverting the tube 2-5 times. The mixture was incubated for $25 \mathrm{mins}$ at $37^{\circ} \mathrm{C}$. Equal volume of phenol chloroform isoamyl alcohol (25:24:1) was added and vortexed vigorously for 20 seconds. The mixture was centrifuged at $10000 \mathrm{~g}$ for $2 \mathrm{mins}$ and supernatant was collected. Then $1 / 10^{\text {th }}$ volume of $0.5 \mathrm{~N} \mathrm{NaCl}$ and 2.5 times ice cool absolute ethanol was added and incubated on ice for 30mins. The solution was centrifuged at $13000 \mathrm{~g}$ for $15 \mathrm{mins}$ at $4^{\circ} \mathrm{C}$ and the supernatant was carefully discarded. The pellet was desalted twice with $70 \%$ ethanol by centrifugation at $13000 \mathrm{~g}$ for 15 mins at room temperature. The ethanol was carefully aspirated, the DNA pellet was air dried and50 $\mu 1$ nuclease free water was added. The purity and concentration of extracted DNA was measured by using agarose gel (1.5\%) electrophoresis and spectophotometry $\left(\mathrm{A}^{260} / \mathrm{A}^{280}\right)$. The concentration of genomic DNA $(100 \mathrm{ng} / \mu \mathrm{l})$ was adjusted by adding nuclease free water. Ratio of $\mathrm{A}^{260}$ and $\mathrm{A}^{280}$ greater than 1.8 was considered as high purity and used in PCR. Appropriate primer sequences for the PCR detection of Meq gene of MD viruses (Table 1) were used as described previously

Table 1.Primers and their sequences used to identify the Meqgene of MD viruses

$\begin{array}{cccc}\begin{array}{c}\text { Primers } \\ \text { name }\end{array} & \text { Primer sequence }\left(5^{\prime}-3^{\prime}\right) & \begin{array}{c}\text { Amplicon size }(\mathrm{bp}) \text { specific } \\ \text { for MD viral strains }\end{array} & \text { Reference } \\ \text { Oligo1F } & \text { tgcgatgaaagtgctatggag } & 185 \mathrm{bp}=\mathrm{JM} / 102 \mathrm{~W} & \text { (Silva et al., } \\ \text { Oligo1R } & \text { gagaatccctatgagaaagcg } & 317 \mathrm{bp}=\mathrm{GA} \text { and } & 1992) \\ & & 449 \mathrm{bp}=\mathrm{Mdll} / 8 \text { strain } & \end{array}$

PCR reactions was performed on each DNA sample in a $25 \mu l$ volume consisting of reaction mixture $(12.5 \mu \mathrm{l})$, primers (forwards and reverse, $25 \mathrm{pmol} / 1 \mu \mathrm{l}$ each), template DNA $(5 \mu \mathrm{l})$ and nuclease free $\mathrm{H}_{2} \mathrm{O}(5.5 \mu \mathrm{l})$. The thermal profile consisted of an initial denaturation for 2 mins at $94^{\circ} \mathrm{C}$ followed by 35 cycles of DNA amplification reaction in a Master Cycler (Master Cycler Gradient, Eppendorf, Germany). The condition of PCR amplifications were denaturation for $60 \mathrm{secs}$ at $94^{\circ} \mathrm{C}$, annealing for $60 \mathrm{secs}$ at $55^{\circ} \mathrm{C}$ and extension for $3 \mathrm{mins}$ at $72^{\circ} \mathrm{C}$ followed by a final extension for $5 \mathrm{mins}$ at 
$72^{\circ} \mathrm{C}$. The PCR reactions were held at $4^{\circ} \mathrm{C}$ and the reaction was terminated by adding $3 \mu 150 \mathrm{mM}$ EDTA. The PCR amplicons were analyzed by electrophoresis in $1.5 \%$ agarose gel, stained with ethidium bromide and examined under UV light using an image documentation system (Cell Biosciences, Alphalmager HP, USA).

\section{RESULTS}

A pullet farm at Ramu Upazilla, Cox's bazar, Bangladesh showed progressive weight loss, wing and leg paralysis. Out of 2200 pullet in the farm, more than $35 \%$ chickens appeared ill during the age of 45-58 days, with a morbidity to mortality rate of 3-4\% per day. About 1000 birds were fallen into chronic sickness during the investigation and 10 sick birds submitted to the Department of Pathology were examined for the clinical signs, pathology, impression smear staining and PCR detection of the specific cause of illness.

\section{Examination of clinical signs}

All of the pullet submitted for necropsy showed either wing or leg paralysis or both (Figure 1). Clinical signs typically observed were depression or unwilling to move, leg dragging/knuckling, drooping of wings, mild opisthotonous and wing and leg paralysis. The characteristics leg paralysis (one leg stretched forward and the other backward) typically involved in Marek's diseases (MD) of chickens was not seen but the leg of infected pullet showed paralysis of its toes (Figure 1a). The flaccid paralysis or paresis of necks and wings were commonly observed. Upon examination of body condition, the birds appeared much thinner with a prominent keel bone (Figure 1b).
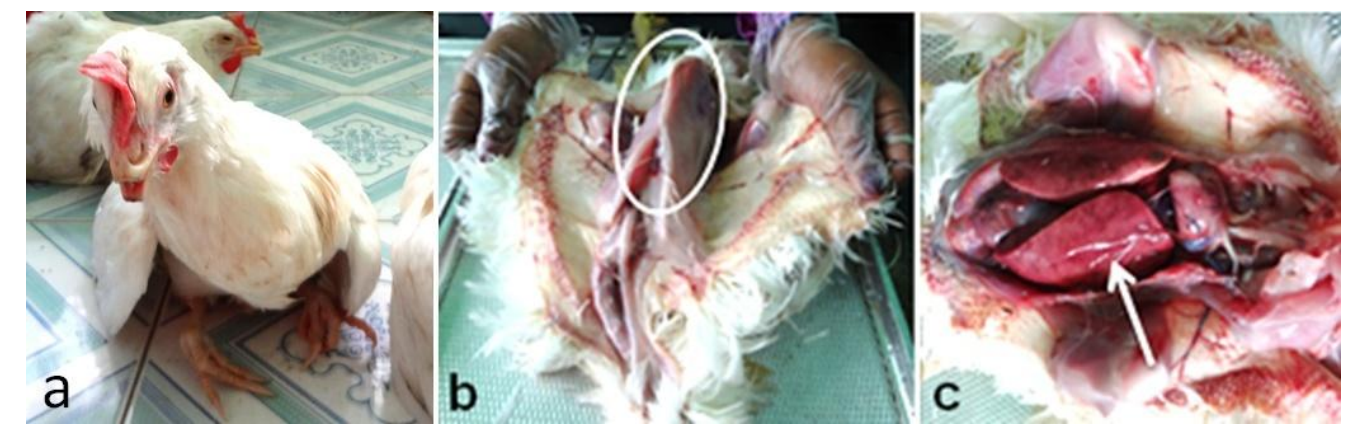

Figure 1. Infected pullets showed paralysis of toe and wings (a), severe atrophy of muscle (b, prominent kill bone) and enlargement of liver (c).

\section{Necropsy findings}

The pullet at necropsy showed very thin musculature and prominent keel bone (Figure 1b). The sciatic (Figure 2a) and brachial nerves were enlarged, the nerve appeared two or three times larger than normal thickness. The infected sciatic nerve 
lost their striation and dull in appearance. Sciatic nerve enlargement was seen in five out of eight chickens necropsied. The liver (Figure 1c and 2d), kidney (Figure 2b) and spleen (Figure 2c) were enlarged with grayish discoloration. Lymphomas as seen in the classical form of MD, was seen as small, soft, grey tumors in the kidney and liver. The feather follicles of infected chickens were severely congested and appeared prominent. Characteristics nodular lesions and enlargement of liver, kidney and spleen of infected chickens were variably noted.

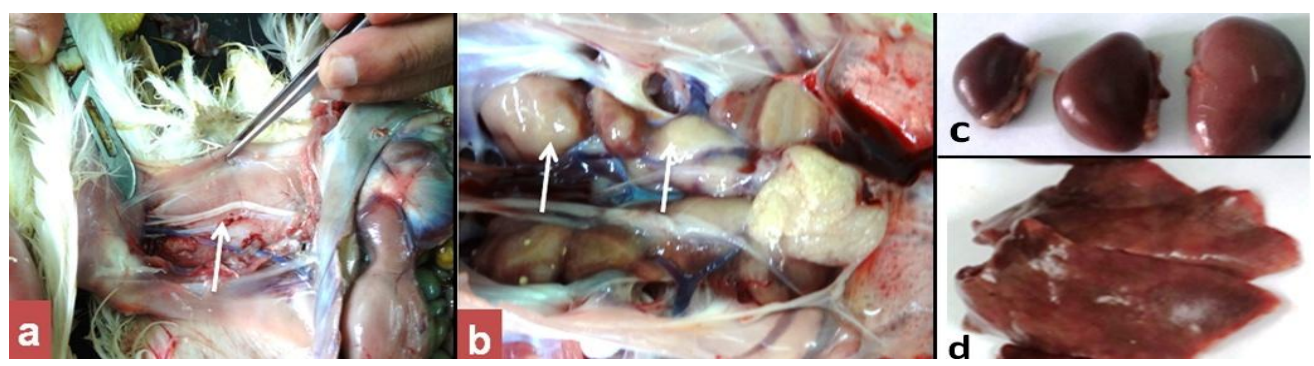

Figure 2. Sciatic nerve of infected pullet appeared distended (a, arrow).There was nodular outgrowth in kidney (b). The splenomegaly (c) and hepatomegaly (d) were observed in 50-60\% of infected chickens.

\section{Histopathologic investigation of affected tissues}

Following histopathological examination, lymphocytic infiltration was seen in all of the tissues examined. The feather follicles (Figure 3a), subcutaneous tissues (Figure $3 b$ ) and cutaneous muscle (Figure 3c) found infiltrating with mononuclear cells. The sciatic nerve showed demyelination and infiltration of lymphocytes (Figure $5 b$ ). Lymphocytic infiltration and accumulation was commonly seen in the lungs (4a), kidney (4b), liver (Figure 4c), gizzard, feather follicles, and heart muscle. The proliferating and infiltrating cells were consisted mostly of lymphoblasts (large, medium and smaller type) with fewer macrophages. The nuclei of lymphocytes were much darker and appeared to be neoplastic. The lympho proliferative nature of the disease was further examined by studying smears of blood and liver smears stained with Giemsa's.

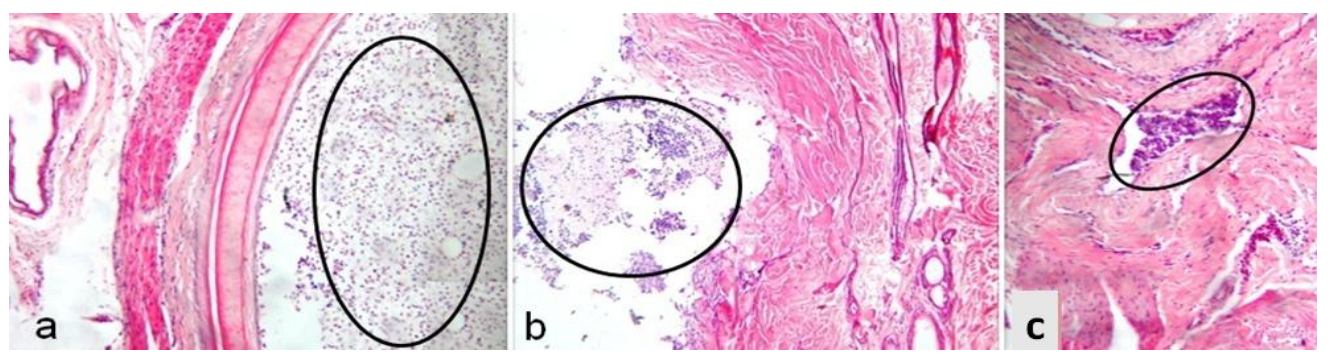

Figure 3. Section of skin from infected pullet stained with H\&E staining. Lymphocytic infiltration and accumulation were seen in feather follicles (a, circle, 10x), subcutaneous tissues (b, circle, 10x) and in cutaneous muscle (c, circle, 40x). 


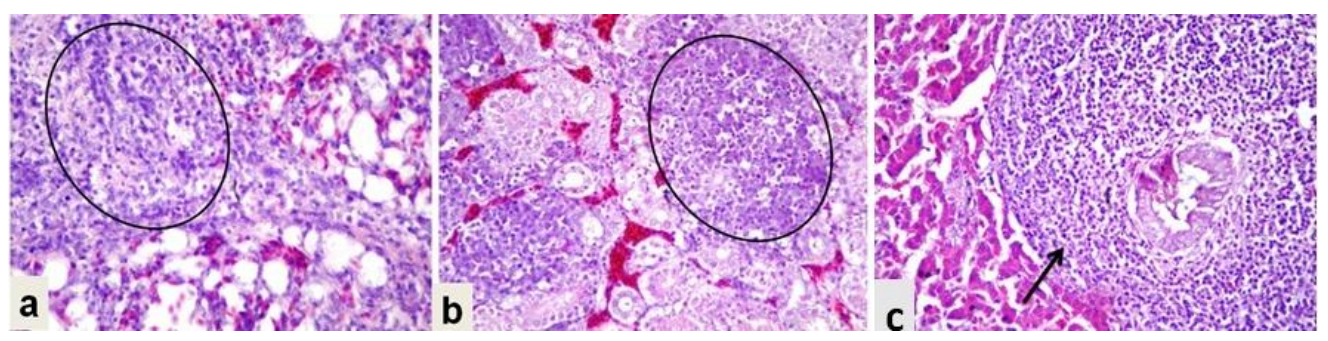

Figure 4. Sections of lungs (a), kidney (b) and liver (c) obtained from infected pullet and stained with H\&E (10x). There was infiltration and aggregation of lymphocytes in the sections of lungs (a, circle), kidney (b, circle) and liver (c, arrow) as seen under microscopic fields.

\section{Smears staining from the liver and blood}

Giemsa's staining is a classic blood film and impression smear staining technique, identify blood cells distinctly. Giemsa's staining of impression smear from liver (Figure 5b) and blood showed infiltration of polymorphic mononuclear cells predominantly lymphocytes. The nuclei of proliferating lymphocytes appeared much darker and thought to be neoplastic in nature but the actual etiology was not confirmed. The lymphoproliferative nature of the disease was, further confirmed by using polymerase chain reaction (PCR).

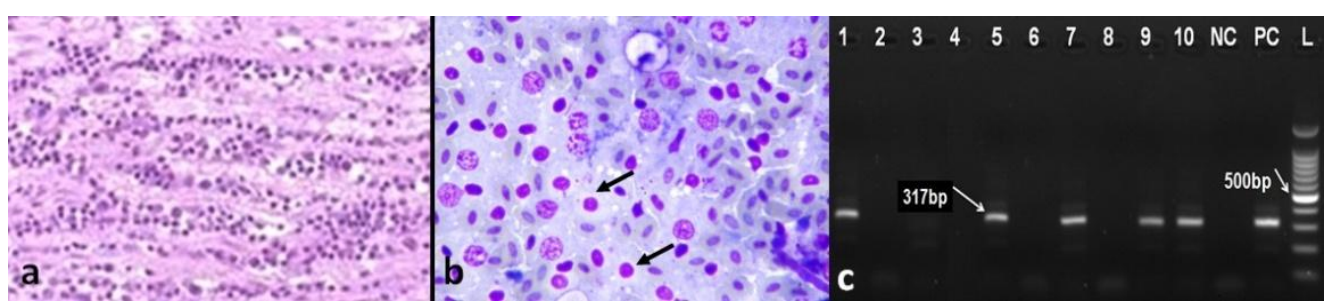

Figure 5. Section of nerve (a) stained with H\&E showed demyelination and infiltration of lymphocytes (10x). Impression smears prepared from the liver (b, 40x) and stained with Giemsa's stain showed huge infiltration of lymphocytes (b, black arrow). DNA extracted from theliver, spleen, heart, kidney, skeletal muscle, feather follicles, sciatic nerve, intestine and lungs were used in PCR amplification of Meq gene of MD viruses. The lane $\mathrm{L}$ is for 100bp ladder, $\mathrm{PC}$ is for positive control, $\mathrm{NC}$ is for negative control and lane 1 to 10 are for organ specific detection of MD. A single band of PCR amplicon (317bp) was found to generate with the DNA extracted from the known positive liver (PC), infected liver (lane 10), spleen (lane 9), kidney (lane 7), feather follicles (lane 5) and lungs (lane 1).

\section{PCR detection of MD virus}

PCR amplification of the Meq gene of serotype $1 \mathrm{MDV}$ was performed with the LAMP F3 and B3 primers and generated a 317bp amplicon in gel electrophoresis (Figure 5c). DNA from the liver, spleen, heart, kidney, skeletal muscle, feather 
follicles, sciatic nerve, intestine and lungs were used in PCR. The fragment of Meq gene was amplified from the liver, spleen, kidney, feather follicles, and lungs suggested the infectivity due to MDV-1.

\section{DISCUSSION}

The causative agent of MD is MDV, which is ubiquitous, globally distributed and very common in commercial poultry flocks with an estimated cost to the industry more than US\$ 1-2 billion (Baigent et al., 2007; Morrow and Fehler 2004). Domestic fowls are the natural host of the MDV, but quail, geese and turkey are also affected. In this study infectivity of pullet due to Marek's disease was investigated by clinical, necropsy, histopathology and PCR means and identifies causes of illness and death.

\section{Investigation of outbreak}

The infected birds at early age showed progressive emaciation, in-appetence, leg and wing paralysis. Marek's disease (MD) is a chronic infection and elderly chickens are mostly affected. The nature of MD has changed since the disease was first described. The classical form of MD was seen in elder chickens and was known as "fowl paralysis". It involved leg and wing paralysis often with a typical appearance of one leg stretched forward and another leg backward but this was absent in this study. The toes appeared curling; the legs were unable to carry body weight and put down on to the ground by taking support from her ventral surface of the body. The incomplete unilateral or bilateral wing paralysis was also observed. The flaccid paralysis of neck was observed. The paralysis of neck, legs and wings could be due to lymphocytic infiltration of peripheral nerves, spinal cord and associated ganglia (Marek, 1907) with myelin degeneration. Mortality seen was generally low, about 3-5\% birds (20-25 birds/ day) found dying every day in the pullet farm. The nature of infectivity as seen in the young and infected pullet indicated the infectivity due to very virulent or very virulent plus MD viruses (Afonso et al., 2001). The flocks were vaccinated against MD viruses but still the pullet had severe forms of MD.

\section{Necropsy and histopathology}

Marek's disease is characterized by $\mathrm{T}$ cell lymphomas and infiltration of nerves and visceral organs by lymphocytes (Angamuthu et al., 2012). Following natural infection, microscopic lesions were observed after one to two weeks, butgross lesions were observed after three to four weeks of infection (Abreu et al., 2016). The pullet showed signs of infectivity at the age of 45 and onwards, hence they may catch the infection at early age (25-30 days of age). The birds at necropsy showed thin musculature, prominent keel bone, congested and inflamed feather follicles, enlarged kidneys, hepatomegaly, splenomegaly, enlargement of sciatic and brachial nerve. The cross striation was absence in the enlarged nerve. The lungs appeared congested and consolidated. Out of 10pullet necropsied tumor growth was seen in the liver $(\mathrm{N}=06)$, spleen $(\mathrm{N}=04)$, kidney $(\mathrm{N}=05)$ and proventriculous $(\mathrm{N}=02)$. Combined splenomegaly and hepatomegaly was seen in three cases. Enlargement of sciatic nerve was seen in 
five cases. A congested and edematous feather follicle was seen in three cases. Out of 10 birds examined, typical lesions suggestive for lympho-proliferative disease were observed in four cases. However, the lesions observed at necropsy such as enlargement of liver, spleen, kidney, nerve were not consistent in all cases. This disease was suspected as a case of MD and the nature of cells infiltrated in visceral organs were further evaluated by using $H \& E$ staining of tissues sections and Giemsa's staining of smears.

\section{Staining of sections and smears}

Staining of tissue sections and smears contribute better information about the cellular type involved in lympho-proliferative disease of chicken and to support diagnosis. Sections of visceral organs stained with $H \& E$ and yielded specific morphology of infiltrated cells. Massive infiltration and accumulation of lymphocytes was seen in lungs, liver, kidney, spleen, nerves, skins and muscles etc. Smears from the liver and blood stained with Giemsa's showed highest densities of lymphocytes followed by macrophages and plasma cells.

Lymphomas in the visceral organs and nerve as seen cytologically were mixed cell types; consisting of small and medium lymphocytes, but in some lesions large lymphocytes and lymphoblasts was also seen. The heterogeneous population of lymphoid cells as seen in $\mathrm{H} \& \mathrm{E}$ stained sections and in impression smears stained with Giemsa's, showed an important feature in differentiating the disease from lymphoid leukosis; in which the lymphomatous infiltrations are composed of uniform lymphoblasts. In lymphoid leukosis, gross lymphomas occur in the bursa of Fabricius, and the tumor has an intra-follicular in origin (Barrow et al., 1999). In MD, although the bursa is sometimes involved in the lympho-proliferation, the tumor is less apparent, diffuse and inter-follicular in location. Peripheral nerve lesions are not a feature of lymphoid leukosis but the lesion is predominated in MD (Renz et al., 2012).

\section{Specific detection of MD by using PCR}

Genes which are unique for MDV-1 strains have been used in attempt to identify and pathotype MDV-1 strains by Using PCR. One major gene of interest is the Meq (MDV EcoRI-Q) gene. The Meqgene is constantly expressed through latency and in all tumors (Kung et al., 2001; Liu et al., 1999). Meq gene has got an anti-apoptotic function and the potential to stimulate cell growth and transformation by MDV-1 (Liu et al.,1999; Renz et al., 2012).In this study PCR amplification was carried out targeting fragment of $M e q$ gene with the DNA extracted from liver, spleen, heart, kidney, skeletal muscle, feather follicles, sciatic nerve, intestine and lungs of infected pullets. DNA extracted from the liver, spleen, kidney, feather follicles and lungs found to generate expected (317bp) amplicon.

In this study a PCR was adapted using published primers (Silva et al., 1992). The PCR primers were designed from the flanking region of MDV1. The amplified band 
(132bp repeat) generated from the flanking region and $185 \mathrm{bp}, 317 \mathrm{bp}$ and $449 \mathrm{bp}$ were specific for pathogenic strain of JM/102W, Md/5 (attenuated MDV1 GA strain) and Mdll/8 viruses, respectively. In this study the amplicon generated was $317 \mathrm{bp}$, suggestive for infectivity with Md/5 (MDV1 GA strain) viruses. The Marek's disease virus involved with the disease processes was belonging to very virulent strain (OIE 2010) as the PCR detected only $317 \mathrm{bp}$ fragment. The attenuated or avirulent strains of MDV1 usually generated more than one band (185bp repeat) in PCR but which was not seen in this study, so the viruses detected were belonging to virulent group. The Md/5 or MDV1 (GA strain) is a very virulent strain of MD virus (OIE, 2010) the viruses also causes higher rate of morbidity and mortality. To enable systematic virulence classification of MDV isolates on the basis of the pathology (Renz et al., 2012) they induce, and their ability to overcome the effects of vaccination, a standardized MDV pathotyping scheme was developed by the USDA ADOL may be used. Virulent MDV1 challenge strains were graded according to the protection induced by HVT and HVT/ MDV2 bivalent vaccines (Witter 1997; Witter et al., 2005). While MD vaccines reduce virus replication and prevent tumor formation, they do not induce sterilizing immunity; vaccinated chickens are still susceptible to infection with pathogenic strains of MDV that replicate in the host and shed into the environment in the absence of clinical disease. The inability of MDV vaccines to prevent virus replication following field challenge ("imperfect vaccination") is thought to have contributed to the worldwide increase in the virulence of MDV over the last 40 to 50 years, as has been postulated as a probable cause of change for other pathogens (Gandon et al., 2001; Renz et al., 2012). This is for the first time in Bangladesh reported the occurrence of very virulent Md/5 (MDV1 GA strain) infectivity in pullet with higher rate of morbidity and mortality. Until 2010 (OIE 2010), the viruses were not approved by the USDA to use commercially in poultry farms. The $\mathrm{Md} / 5$ strain of viral vaccine may be used in our poultry industry somewhere in the globe and thus the virus may have introduced in the pullet farms.Due to limitation of research resources such virulent or pathotyping (Witter et al., 2005) was not carried out in this study.

\section{CONCLUSION}

The pullet in the farm was vaccinated against MDV but still had the clinical outbreaks and mortality due to MD. The etiology of the disease was thought to be due to MDV1GA (Md/5) strain of MDV. This PCR protocol used can be a suitable tool to selectively identify various strains of MSV in a reaction. It needs to isolate the viruses in culture, identify pathotype in vivo and evaluate protective efficacy of the existing viral vaccines against circulating viruses.

\section{ACKNOWLEDGEMENT}

Thanks are due to PIU/BARC/NAPTH-Phase 1 for funding the research 


\section{REFERENCES}

Abreu, D.L.C., Santos, F.F., José, D.S., Tortelly, R., Nascimento, E.R., and Pereira, V.L.A. (2016).Pathological Aspects of a Subclinical Marek's Disease Case in Free-Range Chickens. Brazilian Journal of Poultry Science, 18,197-200.

Afonso, C.L., Tulman, E.R., Lu, Z., Zsak, L., Rock, D.L., and Kutish, G.F. (2001).The genome of turkey herpesvirus. Journal of Virology, 75, 971-978.

Angamuthu, R., Subasty, B., Gopal, D.R., Devarajan, J., and Kathaperumal, K. (2012). Rapid Detection of the Marek's Disease Viral Genome in Chicken Feathers by LoopMediated Isothermal Amplification. Journal of Clinical Microbiology, 50, 961-965.

Baigent, S.J. (2006). Herpes virus of turkey reconstituted from bacterial artificial chromosome clones induces protection against Marek's disease. Journal General Virology, 87, 769776.

Baigent, S.J., Smith, L.P., Currie, R.J., and Nair, V.K. (2007).Correlation of Marek's disease herpesvirus vaccine virus genome load in feather tips with protection, using an experimental challenge model. Avian Pathology, 364, 67-474.

Barrow, A., and Venugopal, K. (1999). Molecular characteristics of very virulent European MDV isolates. Acta Virology, 43, 90-93.

Biggs, P.M. (1961).A discussion on the classification of the avian leucosis complex and fowl paralysis. British Veterinary Journal, 177, 326-334.

Delaney, D.B., Morrow, C.J., Read, K.M., and Tannock, G.A. (1998). The development and evaluation of two tissue culture-grown Marek's disease challenge viruses. Avian Pathology, 27, 472-477.

Gandon, S., Mackinnon, M.J., Nee, S., and Read, A.F. (2001). Imperfect vaccines and the evolution of pathogen virulence. Nature, 414, 751-755.

Handberg, K.J., Nielsen, O.L., and Jorgensen, P.H. (2001).The c polymerase chain $\square$ use of serotype 1 and serotype 3 specific reaction for the detection of Marek's disease virus in chickens. Avian Pathology, 30, 243-249.

Haq, K., Schat, K.A., and Sharif, S. (2013). Immunity to Marek's disease: where are we now? Developmental and Comparative Immunology, 41, 439-446.

Heidari, M., Wang, D., Delekta, P., and Sun, S. (2016). Marek's disease virus immune suppression alters host cellular responses and immune gene expression in the skin of infected chickens. Veterinary Immunology and Immunopathology, 180, 21-28.

Islam, A., Harrison, B., and Cheetham, B.F.(2004). Differential amplification and quantitation of Marek's disease viruses using real-time polymerase chain reaction. Journal of Virological Methods, 119, 103-113.

Islam, A., Harrison, B., Cheetham, B.F., Mahony, T.J., Young, P.L., and Walkden-Brown, S.W. (2004).Differential amplification and quanti-isolates by the "best fit" pathotyping assay. Avian Pathology, 36, 135-143.

Kung, H.J., Xia, L., Brunovskis, P., Li, D., Liu, J.L., and Lee, L.F. (2001). Meq: an MDVspecific bZIPtransactivator with transforming properties. Current Topics in Microbiology and Immunology, 255, 245-260. 
Liu, J.L., Lin, S.F., Xia, L., Brunovskis, P., Li D., Davidson, I., Lee, L.F., and Kung, H.J. (1999). MEQ and V- IL8: cellular gene in disguise? Acta Virologica, 43, 94-101.

Luna, L.G. (1968).Manual of the histologic staining methods of the armed forces institute of pathology. $3^{\text {rd }}$ ed. McGraw Hill Book Society, New York.

Marek, J. (1907).Multiple Nervenenetzuendung (polyneuritis) beiHuehnern. Deutsche Tierarztliche Wochenschrift, 5, 417-421.

Morrow, C., and Fehler, F. (2004).Marek's disease: a worldwide problem. In Marek's disease: an Evolving Problem (eds. Fred Davison and Venugopal Nair).Elsevier Academic Press.

OIE International des Epizooties. (2010).Version adopted by the world assembly of delegates of the OIE, chapter 2.3.13.

Pappenheimer, A.M., Dunn, L.C., and Cone, V. (1929).A study of fowl paralysis (neurolymphomatosis gallinarum.I ) clinical features and pathology. Journal of Experimental Medicine, 49, 63-86.

Payne, L.N., and Venugopal, K. (2000). Neoplastic diseases: Marek's disease, avian leukosis and reticuloendotheliosis. Reviews of Science and Technology, 19, 544-64.

Quere, P. (1992).Suppression mediated in vitro by Marek's disease virus-transformed Tlymphoblastoid cell lines: effect on lymphoproliferation. Veterinary Immunology and Immunopathology, 32,149-164.

Renz, K., Cooke, J., Cheetham, B., Hussain, Z., Islam, A., Tannock, G., and Walkden, B.S.(2012).Pathotyping of Australian isolates of Marek's disease virus and association of pathogenicity with meqgene polymorphism. Avian Pathology, 41, 161-176.

Silva, R.F. (1992). Differentiation of pathogenic and non-pathogenic serotype 1 Marek's disease viruses by the polymerase chain reaction amplification of the tandem direct repeats within the MDV genome. Avian Diseases, 36, 521-528.

Witter, R.L. (1997). Increased virulence of Marek's disease virus field isolates. Avian Diseases, 41,149-163.

Witter, R.L., Calnek, B.W., Buscaglia, C., Gimeno, I.M., and Schat, K.A. (2005). Classification of Marek's disease viruses according to pathotype: philosophy and methodology. Avian Pathology, 34, 75-90.

Zhang, F. (2014).Transcriptional activity comparison of different sites in recombinant Marek's disease virus for the expression of the H9N2 avian influenza virus hemagglutinin gene. Journal of Virological Methods, 207, 138-145. 